\title{
Robots with Biological Brain
}

\section{Ochin* adn Jugnu Gaur}

Faculty of Engineering \& Technology, Manav Rachna International University, Faridabad, India

\begin{abstract}
The term robotics comes from robots. Many governments, commercial and individual bodies are working in the area of Robotics due to its importance in defence, personal and commercial uses. Much research has been put on it, but we could not succeed to a satisfactory level. In this area a small success is also seems as an achievement. But seeing the future commitments of robotics, we are in a need of perfect working robots. Robots are combination of machine plus human intelligence. Where the computer part is concerned; robots are always appreciated as timings of actions are fast; calculations are accurate, remote access is there. But we fail when we see the human part of these robots. Reason is very simple we want to constitute artificial brain into these robots and we cannot constitute something which infect we don't know. When biological science do not claim to have proper in depth working knowledge of brain how we can constitute it with the help of semiconductors and capacitors. Well, indeed it is a problematic challenge that must be sorted out. With this paper I am proposing the idea of combining robotics with biological brains, as artificial DNA's are recently added into our inventions and recent biology is not far away to produce artificial brains.
\end{abstract}

Keywords: Robotics; Artificial biological brain; Robots; Artificial intelligence

\section{Introduction}

Since a long there are efforts from different cultures to create artificial men or robots those have human capabilities but those follows the orders of nature created humans. But even following the orders and accomplish the task/ work requires aptitude and intelligence. Artificial intelligence (AI) is arguably the most exciting field in robotics. It's certainly the most provocative: Everybody agrees that a robot can work in an assembly line, but there is no consensus on if a robot can be intelligent. Ultimate AI would be a regeneration of the human thought process -- a manmade device with our intellectual capacities. This would take in the ability to study just about anything, the ability to aim, the ability to use language and the ability to express original ideas. Roboticists are nowhere near achieving this level of artificial intelligence, but they have made a lot of progress with more limited AI. Today AI machines can imitate only some specific elements of intellectual capacity. Computers can now resolve problems in partial realms. The basic idea of AI problem-solving is very simple, though its execution is complex. The AI robot or computer collects facts about a position through sensors or human effort. The computer relates this information to stored data and chooses what the information signifies. The computer runs through various stored likely actions and forecasts which action will be most suitable based on the composed information. Of course, the computer can only resolve difficulties. Chess computers are one instance of this type of machine. Some recent robots also have the skill to learn in a limited capacity. Learning robots identify if a certain action (for instance moving its legs in a specific manner) attained a desired result (navigating an obstacle). The robot holds this information and attempts the suitable action the next time it meets the same situation. Second, modern computers can only do this in very specified situations. They cannot grip any sort of information like a human can. Few robots can learn by imitating human actions. In Japan, roboticists have taught a robot to dance by demonstrating the moves themselves. Infect, The real challenge of AI is to understand how natural intelligence works.

\section{Robots efficiency as a machine}

Comparing the efficiency of a robot with a machine/mechanical device, a robot proves equivalent good in working with a little intelligence, however no doubt costlier than a machine. Robots perform applications with greater accuracy, precision and consistency. The product quality improves because of these increases. See when comparing with machines robots have always an upper hand because robots are intelligent than a mechanical machine [1].

\section{Operation in unsafe environs}

There are many industries where the surroundings are unsafe for the employ of human labor due to the existence of dangerous environments. Robots can be utilized effectually in such environments where conduct of radioactive materials is involved, such as hospitals or nuclear establishments, where straight exposure to human beings can be unsafe for their health.

\section{Robots in treatment}

Robots are critical to the medical field where great precision and delicacy is necessary, and the edge for mistake slim. Let's see a few subareas:

\section{Surgery}

Robots are capable to perform key operations while only making small incisions, patients obtain many benefits: lessened trauma, fewer infections, less healing time, and a earlier discharge from the hospital. Robots perform heart surgery without opening patients chests.

*Corresponding author: Ochin, Faculty of Engineering \& Technology, Manav Rachna International University, Faridabad, India, E-mail: ochin.fet@gmail.com

Received July 22, 2011; Accepted July 30, 2011; Published July 31, 2011

Citation: Ochin, Gaur J (2011) Robots with Biological Brain. J Comput Sci Syst Biol 4: 042-046. doi:10.4172/jcsb.1000074

Copyright: @ 2011 Ochin, et al. This is an open-access article distributed under the terms of the Creative Commons Attribution License,which permits unrestricted use, distribution, and reproduction in any medium, provided the original author and source are credited. 


\section{Academics}

Robots are now used to test medical students. Pregnant humanoid robots, for instance, prepare students for various birth difficulties.

\section{Administration}

Robots are also affecting the way hospitals are run and medications dispersed. They make sure hospital visits are shorter and the risk of infection reduced.

\section{Development in quality}

Robots do operations with larger exactitude, confirm uniformity of manufacture due to which rejections are reduced, and reduce damages. Measurements and arrangements of tools being utilized are more correct. Thus, the quality of the product manufactured is better manifold related to the performance by human beings.

\section{Growth in production}

Robots have the talent to work nonstop without pause, dissimilar human labor for which breaks and holiday are vital. Thus, manufacturing is increased by the use of robots in industrial applications, and consequently profits of the manufacturing unit are increased.

\section{Execute boring and cyclic work}

In numerous production establishments work essential to be performed is awfully boring, being cyclic and boring, due to which it is tough for the machinists to remain fully devoted to their jobs and generate attentiveness in their work. When tasks are monotonous, employees incline to be careless, thereby growing the likelihood of mishaps and breakdowns of machines. Use of robots has removed problems associated with boredom in manufacturing.

\section{Responsibility during adverse times}

Most of the manufacturing units are necessary to function twenty four hours, on day off, without any break so as to ensure enlarged production which is commensurate with the ability of the machinery. Thus, humans who do not sound very comfortable working such odd hours can be employed therefore. However, robots can be helpfully utilized whenever necessary.

\section{Protection and well-being of workers}

From the time when robots are capable of working in such environments, more dangerous operations are being handled by robots. Thus the safety and well-being of workers is sured, thereby decreasing overheads on health and medicines. Robots are now engaged in hoisting and shifting heavy objects, and accomplish other unsafe tasks.

\section{Robots efficiency as a human vs machine}

When we compare a robot against a human. Here robotics lacks due to humans are more intelligent than robots so a robot lacks in decision making, learning from mistakes or experiences, act differently to the same inputs and many more. So even an animal can act better in realistic environment than this specific machine [2].

In spite of the very useful set of benefits of robotics conversed above, there are some tasks for which human beings are superior to robots. For example:

- Robots are not appropriate for creativity or innovation

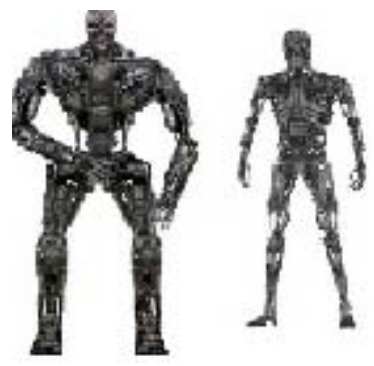

Figure 1: Robots

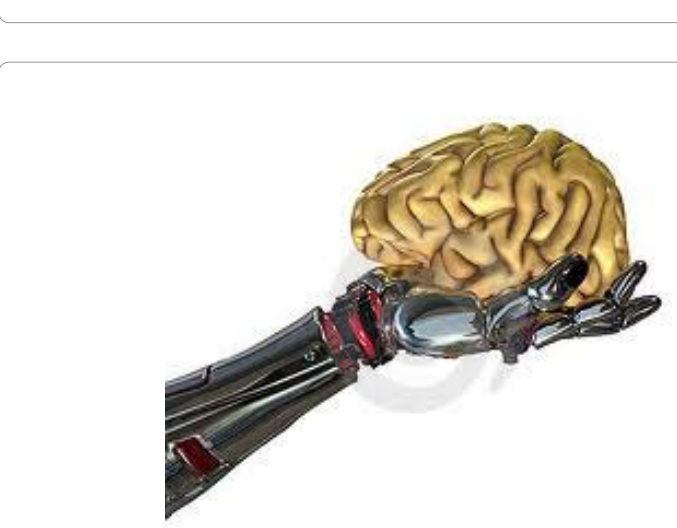

Figure 2:

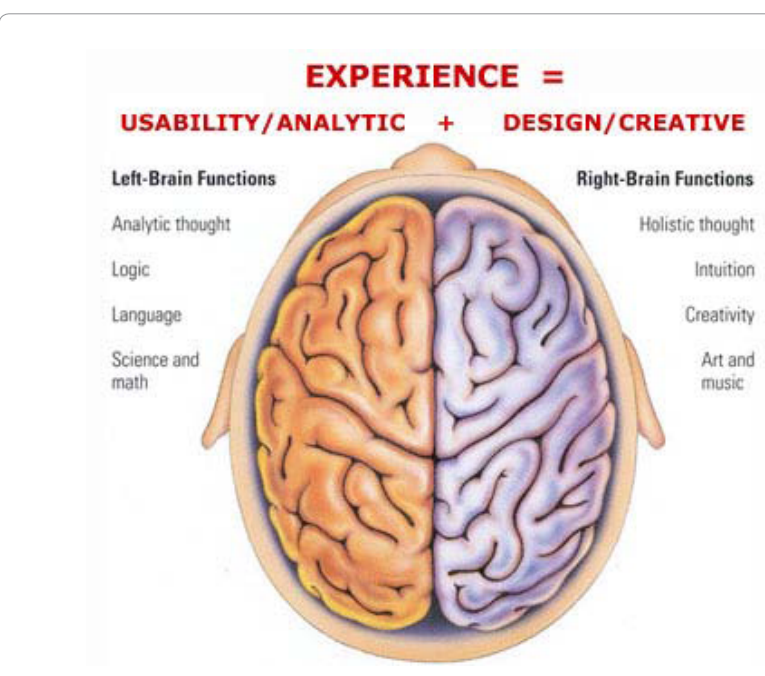

Figure 3:

- Robots don't have ability of independent thinking

- Robots are not good enough to learn from their past experiences.

- Robots are not as appropriate for making difficult decisions

- Robots can't as voluntarily adapt quickly with changes in the environments.

Human beings are required for these sorts of tasks, so there is expectation that we will not become superfluous in a world ruled by robots at some point in the future, as predicted by some science fiction authors! 
Artificial brain is not a suitable or reasonable answer. Since decades we are trying to apply this concept. But all in vein or a very little success we got. Because we cannot produce something whose functionality we exactly don't know. This statement is application in the production of artificial brains also.

\section{Unknown biological brain}

Functionalities: As stated we cannot produce something whose functionality we exactly don't know. Most theologists believe that a mind is supernatural and cannot be reproduced by machines [3].

Memory stored and retrieved: When you cram a new fact, like anyone's name, there are physical changes in the structure of your

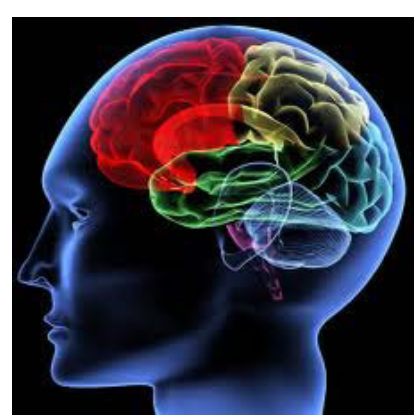

Figure 4:

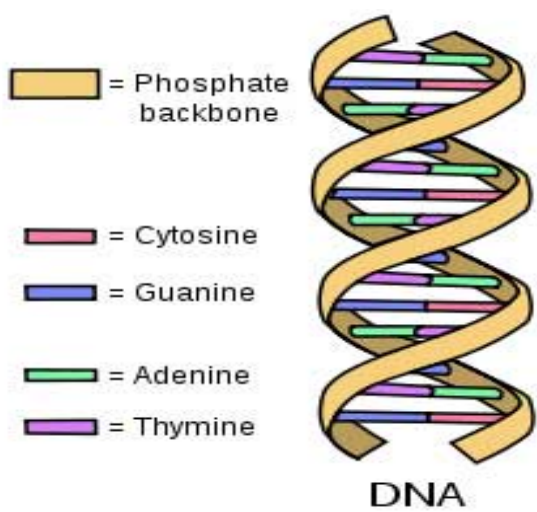

Figure 5:

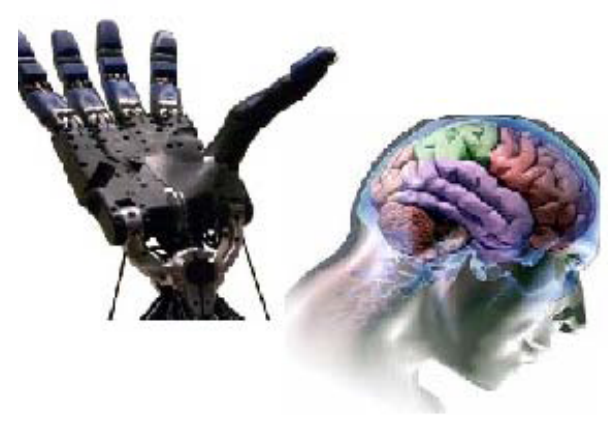

Figure 6: brain. But we don't yet understand exactly what those deviations are, how they are orchestrated crossways vast seas of synapses and neurons, how they embody information, or how they get back decades later for retrieval. One difficulty is that there are numerous kinds of memories. The brain seems to differentiate short-term memory (memorizing a phone number just long enough to dial it) from long-term memory (what you have done on your last birthday). Inside long-term memory, declarative memories (like names and facts) are different from nondeclarative memories

However, same molecular mechanisms may be at work in these memory types. Almost all theories of memory suggest that memory room depends on synapses, the tiny networks between brain cells. When two cells are dynamic at the same time, the connection between them strengthens; when they are not active at the same time, the connection fades. Out of such synaptic changes emerges an relationship. For example, fortify the links between the smell of coffee, taste, color, and the feel of its warmth. Since the neurons connected with each of these sensations are typically activated at the same time, the networks between them can cause all the sensory relations of coffee to be triggered by the smell alone.

Memory retrieval is even more secretive than storage. When I ask if you know Mr. Amit, the answer is instantly obvious to you, and there is no black and white theory to clarify how memory retrieval can occur so rapidly.

Baseline activity in the brain represents: Neuroscientists have generally considered changes in brain movement that correlate with stimuli we can present in the laboratory, such as a picture, a touch, or a sound. But the movement of the brain at rest-its "baseline" activity-may show to be the most significant aspect of our cerebral lives. Resting brain uses 20 percent of the body's total oxygen, although it contributes only 2 percent of the body's mass. A number of the baseline activity may signify the brain restructuring information in the background, simulating future states and events, or operating memories. Most things we care about reminiscences, emotions, drives, plans; can happen with no external stimulus and without measured output.

Remember that sensory input is excessive for perception: When your eyes are shut during dreaming, you still enjoy rich visual involvement. The awake state may be basically the same as the dreaming state, only somewhat anchored by external stimuli. In this view, can I say your conscious life is an awake dream.

\section{How brains do predicts the future}

We distinguish little about how the brain's future simulator works as previous neuroscience technologies are best suited for correlating brain activity with explicit behaviors, not mental imitations. One idea proposes that the brain's resources are devoted not only to processing stimuli and responding to them (viewing a ball come at you) but also to structure an interior model of that outside world and extracting rules for how stuffs tend to work (knowing how balls move over the air). Interior models may show a role not only in motor acts, but also in perception.

\section{What are emotions}

Present views suggest that emotions are brain states that rapidly assign value to outcomes and deliver a simple plan of act. Hences, feeling can be viewed as a type of computation, a quick, automatic 
summary that starts suitable actions. When a tiger is galloping toward you, the rising fear directs your brain to do the right things (finding an escape route) instead of all the other things it could be doing. When it comes to perception, you can spot an object more rapidly. If a spider rather than a roll of tape. In the realm of memory, emotional occasions are laid down contrarily by a similar memory system associating a brain area called the amygdala.

One objective of emotional neuroscience is to get the nature of the many ailments related with emotion, depression is the most common .Impulsive aggression and violence are also thought to be consequences of defective emotion regulation[4].

\section{What is intelligence}

Intelligence originates in many ways but it is unknown what intelligence exactly is biologically. How do billions of neurons work together to employ knowledge, simulate new situations, and remove inconsequential information. What happens in your brain when you suddenly got that the killer in the movie is actually the unsuspected man. Do intelligent people holds knowledge in a mode that is more distilled, more different, or more effortlessly retrievable.

Present experiments explore the likely relationship of intelligence and short term memory, the ability to speedily resolve cognitive conflict, or the ability to store tougher associations between facts and the outcomes are not however conclusive. Several other likelihoods improved restructuring of stored information, more parallel processing, or higher emulation of possible futures have not been probed by experiments.

Intelligence might not be reinforced by a single mechanism or a single neural area [5].

\section{Time representation in the brain}

When it derives to awareness, the brain goes through a worthy deal of trouble to synchronize arriving signals that are processed at very different speeds.

For a simple instance in what way your brain plays tricks with time, look in the mirror at your left side eye. Now shift your gaze to your right side eye. Your eye actions take time but you do not realize your eyes exchange. It is as if the world suddenly made the transition from one view to the other. What occurred to that tiny gap in time? What happens to the 80 milliseconds of darkness you should get every time you blink your eyes? Bottom line: Your view of the flat passage of time is a construction of the brain. Clarifying the picture of how the brain normally solves timing problems should give insight into what occurs when temporal calibration goes wrong, as may happen in the brains of people with dyslexia. Sensory efforts that are out of synchronization also add to the risk of falls in aged patients [6].

\section{Robots with biological brains}

The solution of above problems are to equip robots with biological brains; let the mystery solve the mystery.

Innovation of artificial DNA leads to the biological computers--- It has been 50 years then the discovery of the double-stranded nature of DNA, and more than half-century and more we have learned a lot for deoxyribonucleic acid, from the fact that it organizes into a doublestranded double helix all the way to having sequenced the complete
DNA of humans and a variety of other organisms. Now, Japan has made the world's first DNA strand made from artificial bases.

As information storing systems go, DNA is not just four different bases (adenine, thymine, guanine, and cytosine), are all that's needed to code for 20 dissimilar amino acids, by three base codons. In fact, the four-base, triplet codon system has the ability for storing information for more than just 20 amino acids; there are 64 possible combinations, so a number of amino acids have several codons, along with three stop codons that tell the cellular mechanism involved that the sequence is done [7].

Moreover, people have observed at DNA and believed that it ought to be potential to use DNA to store non - biological data. Better still, it can pack that fact into far slighter packages than is probable with solid state memory or even the densest hard drive platters. There have also been tests that use DNA patter ns to do concurrent processing.

But we need not be narrow to the four paired bases, and thisis just what has been shown by a Japanese team, who have issued details of their creation of an artificial DNA strand. All the mechanisms of their DNA product are non-natural, yet they form right-handed duplexes with the corresponding contrasting base, and these bonds have very similar belongings to those of natural DNA [7].

The optimism is that this artificial DNA might have a choice of applications in the physical world, from the said DNA computing proposals, along with expending DNA to store data, to use it in nanotech settings. Artificial DNA has alike physical properties to common or garden DNA without being degraded by enzymes such as DNase (which is found universally), an aspect that would make it pretty useful for any type of biomedical setting.

Also, tests are going on to improve biological brain with the help of cultured rat neurons. The simple mechanism follows by attaching a layer of proteins to a silicon disk and to add brain cells of embryonic rats that bind themselves to the proteins and nurtured to connect with one another in a ring.

It would be worth saying that the "multi-electrode array" (MEA) can play a key role of an interface between live tissue and device with the organic brain emitting electrical impulses for movement in the wheels of the robot, and receiving impulses carried by sensors reacting to the medium.

Because of the fact that the brain is made of living tissue, scientists collect it in an exceptional unit where scientists can maintain the level of temperature. It can link with the robot's body by a Bluetooth radio link.

Considering this as an example, man is going closer to what he is aiming in respect of AI however the effect was a continual redrawing of the margin between human and machine and redefining the essence of life and intelligence.

\section{Conclusion}

We know we cannot separate AI and robotics but applying almost utmost technologies collectively since decades in this regard, we are unable to get the results what was expected. The reason is brain intelligence is a vital factor of humans. To allow robots to become intelligent, we tried to develop something that compensates the 
brain organ in robots. But actually till date we are not sure the entire working of brain how we can manufacture it merely using electronics devices. So this is the time of rebuilding the concept. By utilizing the development of other branches say biology, we can use the biological brain to equipped our robotics field with the intelligence, decision making, and new learning capacity.

\section{References}

1. http://machineslikeus.com/news/machines-may-soon-argue-back-humans

2. http://machineslikeus.com/news/when-brain-remembers-patient-doesnt
3. Mitterauer B, Kopp K (2003) The self-composing brain: Towards a glialneuronal brain theory. Brain Cogn 51: 357-367.

4. A Working Brain Model. Source: Complexity Digest. Frontiers in Neuroscience 2: 6-9.

5. The Four Quadrant Model of the Brain, Ned Herrmann's Whole Brain Model.

6. The Reptilian Brain and the Triune Brain Model (1/7).

7. http://www.telegraph.co.uk/health/healthnews/5771252/Scientists-createartificial-brain-cell.html 